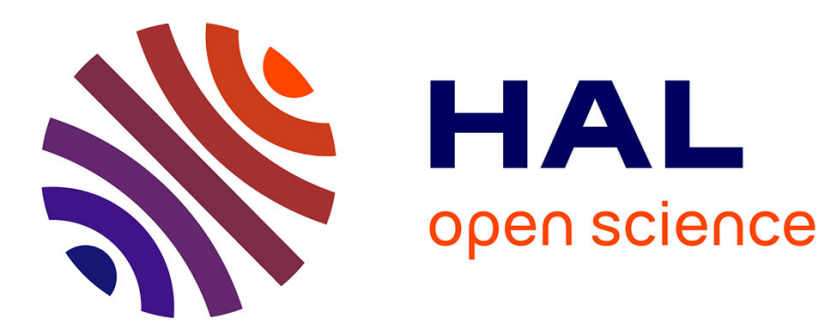

\title{
Experiments on the CEC 2015 expensive optimization testbed
}

\author{
Vincent Berthier
}

\section{To cite this version:}

Vincent Berthier. Experiments on the CEC 2015 expensive optimization testbed. CEC 2015

- IEEE Congress on Evolutionary Computation, May 2015, Sendai, Japan. pp.1059 - 1066 10.1109/CEC.2015.7257007 . hal-01215805

\section{HAL Id: hal-01215805 \\ https://inria.hal.science/hal-01215805}

Submitted on 6 Nov 2015

HAL is a multi-disciplinary open access archive for the deposit and dissemination of scientific research documents, whether they are published or not. The documents may come from teaching and research institutions in France or abroad, or from public or private research centers.
L'archive ouverte pluridisciplinaire HAL, est destinée au dépôt et à la diffusion de documents scientifiques de niveau recherche, publiés ou non, émanant des établissements d'enseignement et de recherche français ou étrangers, des laboratoires publics ou privés. 


\title{
Experiments on the CEC 2015 expensive optimization testbed
}

\author{
Vincent Berthier \\ TAO, Inria, Univ. Paris-Sud, UMR CNRS 8623 \\ Bat 660 Claude Shannon Univ. Paris-Sud, 91190 Gif-sur-Yvette, France \\ Email: \{firstname.lastname\}@inria.fr
}

\begin{abstract}
We experiment various simple classical algorithms on the expensive optimization testbed from Cec2015. CMA performs best, in particular its DCMA flavor using quasi-random numbers. Nelder-Mead also performs well. Portfolios performed well for the given budget (500 evaluations in dimension 10 and 1500 evaluations in dimension 30), but not the half budget, which is also taken into account in the competition, hence we did not include them in the final version.
\end{abstract}

Keywords-Expensive, Quasi-Random, Optimisation

\section{INTRODUCTION}

[1] proposed a testbed for expensive optimization. This means that the number of fitness evaluations is limited. In this case the budget is 500 in dimension 10 and 1500 in dimension 30 . The testcase is also designed for difficult objective functions, with 2 unimodal and 13 multimodal functions including hybrid and composition functions.

[2] proposed the use of portfolio methods in noise-free optimization. Portfolio are generic tools for combining optimization algorithms, most widely used in combinatorial optimization, but also appearing in noisy continuous optimization[3] and noise-free continuous optimization[2].

We also use quasi-random numbers in mutations, as proposed in [4]. Basically, it makes algorithms slightly better on average, with a different distribution, as detailed later.

The computation time in portfolio algorithms can be simply divided equally among solvers, or not[5]. [6], [7] propose $50 \%$ for the best solver, $25 \%$ for the second best, and so on. One might also run all solvers during e.g. $25 \%$ of total time, and then keep 75\% of the budget only for the best performing one. This is the approach we keep for all our portfolio experiments in this paper.

Surrogate models are classical for expensive optimization[8]; population-based methods have also been widely used[9], as well as derivative-free methods as expensive optimization is often due to heavy simulations without gradient[10]. Gaussian processes are also widely used as their internal cost becomes negligible in front of the cost of the objective function[11], [12]. We consider mostly population-based optimization; the comparison with the results of other methods will be outputs of the session, comparing various methods on this same testbed.

\section{AlgorithmS}

\section{A. Individual algorithms}

We use the following implementation in our comparison ( $N$ is the dimension):

- Covariance Matrix Adaptation (CMA)[13], $\lambda=4+$ $3 \log (N), \mu=\lambda / 2$.

- $\quad$ anisotropic self-adaptive[14], $\lambda=12, \mu=3, \tau=$ $1 / \sqrt{2 N}$.

- $\quad$ Self-adaptive (SA) [14], $\lambda=12, \mu=3, \tau=1 / \sqrt{2 N}$, $\tau_{C}=1+N(N+1) / 2 \mu$.

- $\quad$ Self-adaptive (SA) with covariance[15], $\lambda=12, \mu=$ $3, \tau=1 / \sqrt{2 N}, \tau_{C}=1+N(N+1) / 2 \mu$.

- Differential evolution, DE[16], population size 30, DE/Curr-to-best/1, $C r=.5, F_{1}=F_{2}=.8$.

- Covariance matrix self-adaptation CMSA[17], $\lambda=12$, $\mu=3, \tau=1 / \sqrt{2 N}$.

- $\quad(1+1)$-ES[15], step-size multiplied by 1.5 in case of success and one-fifth rule (i.e. division by $1.5^{1 / 4}$ in case of failure).

- $\quad$ Nelder-Mead[18].

- $\quad \mathrm{PSO}$ [19], [20] with population size 30, neighborhoud $10, \omega=1 /(2 \log (2)), \phi_{p}=.5+\log (2), \phi_{g}=.5+$ $\log (2)$, initial velocity 1 and maximum velocity 1.5 .

The initialization is uniform in the domain, for each algorithm. Due to space restrictions, algorithms are not detailed here, but given references should abswer any question in that regard.

\section{B. Restart/portfolio}

[2] reported excellent results for sophisticated methods, and indeed also good stable results with simple methods. We decided to focus on simple methods. In all our experiments, the portfolio equally divides the computation time among the algorithms during $25 \%$ of the budget, and then uses only the solver which provided the best search point during the remaining $75 \%$ of the budget. CMA/CMA/CMA for example refer to running 3 instances of CMA during 25\% of the budget, and then the best performing one during the remaining $75 \%$.

\section{EXPERIMENTS}

In the following sections, we first compare several classical algorithms, before tuning the best one and adding quasirandom numbers. 


\section{A. Preliminary experiments: comparing various algorithms}

Fig. I, II, III present the results for dimension 10 and 30 for the worst, median and average rank over the test cases.

A short discussion is that CMA and NM perform well, as well as their combinations into portfolios. The next sections are dedicated to testing variants of CMA, in particular including quasi-random numbers and different parametrization.

\section{B. Tuning, and adding quasi-random numbers}

BI refers to initial step-size 40, whereas the initial stepsize is 1 otherwise. QR refers to variants with quasi-random. MS refers to variants with step-size lower-bounded by 0.0001 instead of 0.01 for others. "+" refers to elitist strategies compared to the default "," strategy. Tables IV, V, VI, VII present the results for dimension 10 and 30 for the best, worst, median and average rank over the test cases. The best is usually better with quasi-random numbers, and the worst is worse. Means and medians are usually improved when using quasirandom numbers, though it is not always the case. In Section $\mathrm{V}$, based on means and medians, we get a clear improvement with quasi-random numbers as all best methods use quasirandom mutations. BI usually provides an improvement, as well as the use of a "+" strategy.

\section{CEC 2015 CRITERIA}

The CEC 2015 testbed uses an average between the mean and the best fitness, at the end of the budget of 1500 evaluations (in dimension 30) and 500 evaluations (in dimension 10), and also at half budget. The lowest errors we were able to obtain with one variant of CMA-ES (termed CMASPHI-QR in the following sections) are reproduced in tables VIII and IX. The corresponding complexities are shown in tables $\mathrm{X}$ and XI.

\section{OTHER RESULTS}

In this section, we present several results for all tested algorithms. We reproduced each run 10 times; the standard deviation provided in this section is the standard deviation of the score; i.e. they correspond to the variability of one run. Thanks to the averaging over 10 runs, our results are more significative.

Table XII synthesizes the results of different variants of CMA-ES. HI refers to 80 as an initial step-size. MI refers to 20 as an initial step-size. SP refers to small population size $\frac{1}{2}(3 \log (N)+4)$ instead of $3 \log (N)+4$. BP refers to big population size $2(3 \log (N)+4)$.

Nelder-Mead variants are shown in table XIII. For Differential Evolution (Table XIV), the best performing one is a classical current to best with reasonably standard parametrization. PSO results are shown in table XV. Unless stated otherwise, the initial velocity is 1 and the maximum velocity $\mathrm{MV}$ is 1.5 .

\section{CONCLUSION}

We compared various algorithms on the CEC 2015 testbed. CMA and Nelder-Mead performed best. We validated quasirandom mutations for CMA, i.e. DCMA usually (though not always) outperformed CMA. In particular, all algorithms ranked 1 were equipped with $\mathrm{QR}$ mutations. Our best final combination is a flavor of DCMA, including covariance matrix adaptation and quasi-random numbers. We did not include mirroring[21]. We did not investigate sophisticated memmetic algorithms on top of CMA. We did not experiments on dimensions other than those proposed in the Cec2015 expensive optimization testbed. Portfolios methods were tested, and validated for the total budget, but not for the mid-budget criterion.

\section{REFERENCES}

[1] Q. Chen, B. Liu, Q. Zhang, J. J. Liang, P. N. Suganthan, and B. Y. $\mathrm{Qu}$, "Problem definition and evaluation criteria for cec 2015 special session and competition on bound constrained single-objective computationally expensive numerical optimization," Computational Intelligence Laboratory, Zhengzhou University, China and Nanyang Technological University, Singapore, Tech. Rep., 2014.

[2] P. Baudis and P. Posik, "Online black-box algorithm portfolios for continuous optimization." in Proceedings of PPSN, 2014, pp. 40-49.

[3] M.-L. Cauwet, J. Liu, and O. Teytaud, "Algorithm portfolios for noisy optimization: Compare solvers early," in Proceedings of the 8th Conference on Learning and Intelligent Optimization, 2014.

[4] O. Teytaud and S. Gelly, "Dcma: yet another derandomization in covariance-matrix-adaptation," in GECCO '07: Proceedings of the 9th annual conference on Genetic and evolutionary computation. New York, NY, USA: ACM, 2007, pp. 955-963.

[5] L. Pulina and A. Tacchella, "A self-adaptive multi-engine solver for quantified boolean formulas," Constraints, vol. 14, no. 1, pp. 80-116, 2009.

[6] M. Gagliolo and J. Schmidhuber, "A neural network model for interproblem adaptive online time allocation," in 15th International Conference on Artificial Neural Networks: Formal Models and Their Applications. Springer, 2005, pp. 7-12.

[7] - "Learning dynamic algorithm portfolios," vol. 47, no. 3-4, 2006, pp. 295-328.

[8] M. El-Beltagy, P. Nair, and A. Keane, "Metamodeling techniques for evolutionary optimization of computationally expensive problems: Promises and limitations," in Proc. of the 1999 Genetic and Evolutionary COnference (GECCO 1999), D. G. . al., Ed. Morgan Kaufmann, 1999, pp. 196-203.

[9] C. Poloni and V. Pediroda, "GA coupled with computationaly expensive simulations: tools to improve efficiency," in Genetic Algorithms and Evolution Strategies in Engineering and Computer Sciences. John Wiley, 1997, pp. 267-288.

[10] A. Booker, J. D. Jr., P. Frank, D. Serafini, V. Torczon, and M. Trosset, "A rigorous framework for optimization of expensive functions by surrogates," Structural Optimization, vol. 17, no. 1, pp. 1-13, 1999.

[11] D. R. Jones, M. Schonlau, and W. J. Welch, "Efficient global optimization of expensive black-box functions," J. of Global Optimization, vol. 13, no. 4, pp. 455-492, 1998.

[12] J. Villemonteix, E. Vazquez, and E. Walter, "An informational approach to the global optimization of expensive-to-evaluate functions," Journal of Global Optimization, Submitted.

[13] N. Hansen and A. Ostermeier, "Completely derandomized selfadaptation in evolution strategies," Evolutionary Computation, vol. 11, no. 1, 2003.

[14] H.-G. Beyer, The Theory of Evolution Strategies, ser. Natural Computing Series. Springer, Heideberg, 2001.

[15] I. Rechenberg, Evolutionstrategie: Optimierung Technischer Systeme nach Prinzipien des Biologischen Evolution. Stuttgart: FrommanHolzboog Verlag, 1973.

[16] R. Storn and K. Price, "Differential evolution: A simple and efficient heuristic for global optimization over continuous spaces," J. of Global Optimization, vol. 11, no. 4, pp. 341-359, Dec. 1997. [Online]. Available: http://dx.doi.org/10.1023/A:1008202821328

[17] H.-G. Beyer and B. Sendhoff, "Covariance matrix adaptation revisited - the CMSA evolution strategy," in Proceedings of PPSN, G. Rudolph, T. Jansen, S. M. Lucas, C. Poloni, and N. Beume, Eds., 2008, pp. 123132. 


\begin{tabular}{|c|c|c|c|c|c|c|c|c|c|c|c|c|c|c|c|c|c|}
\hline \multicolumn{18}{|c|}{ Dim 10, worst } \\
\hline & CMA/CMA & \begin{tabular}{|l} 
CMA/ \\
CMA \\
/CMA
\end{tabular} & NM & $\begin{array}{l}\mathrm{DE} / \\
\mathrm{DE} \\
/ \mathrm{DE}\end{array}$ & $\begin{array}{c}\mathrm{NM} / \\
\mathrm{DE} \\
/ \mathrm{PSO}\end{array}$ & CMA & $\begin{array}{l}\text { NM/ } \\
\text { NM } \\
\text { /NM }\end{array}$ & $\mathrm{DE}$ & $\begin{array}{l}\text { CMSA/ } \\
\text { NM } \\
\text { /PSO }\end{array}$ & \begin{tabular}{|c|} 
CMSA/ \\
DE \\
/PSO
\end{tabular} & SAiso & $\begin{array}{l}\mathrm{PSO} / \\
\mathrm{PSO} \\
\text { /PSO }\end{array}$ & SA & \begin{tabular}{|l} 
CMSA/ \\
CMSA \\
/CMSA
\end{tabular} & SAcov & CMSA & PSO \\
\hline 1 & 1 & 3 & 6 & 9 & 10 & 2 & 4 & 11 & 5 & 7 & 8 & 13 & 16 & 14 & 17 & 12 & 15 \\
\hline 2 & 10 & 5 & 3 & 4 & 2 & 7 & 1 & 6 & 14 & 9 & 15 & 16 & 17 & 11 & 12 & 13 & 8 \\
\hline 3 & 1 & 2 & 3 & 6 & 10 & 4 & 9 & 7 & 5 & 14 & 8 & 13 & 15 & 11 & 12 & 17 & 16 \\
\hline 4 & 2 & 10 & 7 & 3 & 1 & 16 & 6 & 5 & 9 & 11 & 15 & 14 & 4 & 17 & 13 & 8 & 12 \\
\hline 5 & 11 & 12 & 1 & 6 & 4 & 16 & 2 & 7 & 13 & 3 & 17 & 14 & 5 & 15 & 10 & 8 & 9 \\
\hline 6 & 1 & 4 & 8 & 9 & 10 & 6 & 5 & 3 & 2 & 11 & 7 & 16 & 13 & 15 & 14 & 12 & 17 \\
\hline 7 & 6 & 2 & 7 & 9 & 4 & 5 & 1 & 10 & 3 & 14 & 8 & 11 & 12 & 15 & 16 & 13 & 17 \\
\hline 8 & 3 & 4 & 1 & 6 & 9 & 2 & 7 & 10 & 5 & 12 & 8 & 14 & 17 & 11 & 15 & 16 & 13 \\
\hline 9 & 9 & 13 & 17 & 2 & 5 & 1 & 16 & 7 & 14 & 11 & 10 & 4 & 6 & 12 & 3 & 15 & 8 \\
\hline 10 & 2 & 9 & 1 & 6 & 3 & 4 & 10 & 5 & 17 & 13 & 15 & 8 & 12 & 7 & 14 & 11 & 16 \\
\hline 11 & 1 & 2 & 3 & 9 & 5 & 4 & 7 & 10 & 6 & 12 & 17 & 11 & 8 & 15 & 16 & 13 & 14 \\
\hline 12 & 5 & 4 & 6 & 2 & 1 & 12 & 3 & 10 & 16 & 8 & 9 & 11 & 15 & 17 & 13 & 14 & 7 \\
\hline 13 & 5 & 2 & 3 & 6 & 9 & 4 & 1 & 12 & 10 & 13 & 8 & 7 & 17 & 11 & 15 & 14 & 16 \\
\hline 14 & 6 & 1 & 4 & 5 & 2 & 7 & 8 & 3 & 12 & 13 & 10 & 16 & 15 & 11 & 9 & 14 & 17 \\
\hline 15 & 4 & 3 & 7 & 1 & 11 & 2 & 16 & 9 & 12 & 8 & 5 & 13 & 10 & 6 & 14 & 15 & 17 \\
\hline avg & $\begin{array}{c}4.5 \\
\pm 0.88\end{array}$ & $\begin{array}{l}5.1 \\
\pm 1\end{array}$ & $\begin{array}{c}5.1 \\
\pm 1.1\end{array}$ & $\begin{array}{c}5.5 \\
\pm 0.7\end{array}$ & $\begin{array}{c}5.7 \\
\pm 0.95\end{array}$ & $\begin{array}{c}6.1 \\
\pm 1.2\end{array}$ & $\begin{array}{c}6.4 \\
\pm 1.3\end{array}$ & $\begin{array}{c}7.7 \\
\pm 0.74\end{array}$ & $\begin{array}{c}9.5 \\
\pm 1.3\end{array}$ & $\begin{array}{c}11 \\
\pm 0.79\end{array}$ & $\begin{array}{l}11 \\
\pm 1\end{array}$ & $\begin{array}{c}12 \\
\pm 0.9\end{array}$ & $\begin{array}{c}12 \\
\pm 1.2\end{array}$ & $\begin{array}{c}13 \\
\pm 0.85\end{array}$ & $\begin{array}{c}13 \\
\pm 0.9\end{array}$ & $\begin{array}{c}13 \\
\pm 0.66\end{array}$ & $\begin{array}{c}13 \\
\pm 0.97\end{array}$ \\
\hline unimodal & 5.5 & 4 & 4.5 & 6.5 & 6 & 4.5 & 2.5 & 8.5 & 9.5 & 8 & 12 & 14 & 16 & 12 & 14 & 12 & 12 \\
\hline multimodal & 4.3 & 5.2 & 5.2 & 5.4 & 5.7 & 6.4 & 7 & 7.5 & 9.5 & 11 & 11 & 12 & 11 & 13 & 13 & 13 & 14 \\
\hline \multicolumn{18}{|c|}{ Dim 30, worst } \\
\hline & $\begin{array}{l}\text { NM/ } \\
\text { NM } \\
\text { /NM }\end{array}$ & NM & CMA & CMA/CMA & \begin{tabular}{|l|} 
CMA/ \\
CMA \\
/CMA
\end{tabular} & $\begin{array}{l}\mathrm{NM} / \\
\mathrm{DE} \\
/ \mathrm{PSO}\end{array}$ & $\mathrm{DE}$ & $\begin{array}{l}\mathrm{DE} / \\
\mathrm{DE} \\
/ \mathrm{DE}\end{array}$ & $\begin{array}{c}\text { CMSA/ } \\
\text { NM } \\
\text { /PSO }\end{array}$ & \begin{tabular}{|c|}
$\mathrm{CMSA} /$ \\
$\mathrm{DE}$ \\
$/ \mathrm{PSO}$
\end{tabular} & SAiso & PSO & $\begin{array}{l}\mathrm{PSO} / \\
\mathrm{PSO} \\
\text { /PSO }\end{array}$ & CMSA & $\begin{array}{l}\text { CMSA/ } \\
\text { CMSA } \\
\text { /CMSA }\end{array}$ & SA & SAcov \\
\hline 1 & 5 & 4 & 3 & 1 & 2 & 7 & 9 & 10 & 6 & 11 & 8 & 12 & 13 & 15 & 14 & 16 & 17 \\
\hline 2 & 1 & 2 & 6 & 11 & 7 & 5 & 4 & 8 & 16 & 10 & 14 & 3 & 9 & 15 & 13 & 12 & 17 \\
\hline 3 & 2 & 4 & 1 & 8 & 9 & 6 & 7 & 5 & 3 & 11 & 10 & 16 & 15 & 13 & 12 & 17 & 14 \\
\hline 4 & 1 & 2 & 8 & 6 & 7 & 5 & 3 & 4 & 14 & 15 & 9 & 10 & 16 & 12 & 11 & 17 & 13 \\
\hline 5 & 1 & 2 & 16 & 14 & 15 & 4 & 11 & 12 & 13 & 3 & 9 & 10 & 7 & 5 & 8 & 6 & 17 \\
\hline 6 & 4 & 1 & 5 & 3 & 2 & 8 & 10 & 9 & 6 & 11 & 7 & 13 & 12 & 17 & 16 & 15 & 14 \\
\hline 7 & 5 & 6 & 2 & 1 & 3 & 8 & 9 & 7 & 4 & 10 & 14 & 11 & 12 & 17 & 13 & 15 & 16 \\
\hline 8 & 4 & 5 & 1 & 2 & 3 & 8 & 9 & 7 & 6 & 10 & 11 & 13 & 12 & 15 & 14 & 16 & 17 \\
\hline 9 & 3 & 5 & 10 & 13 & 15 & 4 & 1 & 9 & 6 & 8 & 11 & 2 & 16 & 14 & 17 & 12 & 7 \\
\hline 10 & 1 & 3 & 5 & 6 & 2 & 8 & 9 & 4 & 12 & 7 & 10 & 14 & 13 & 11 & 17 & 15 & 16 \\
\hline 11 & 4 & 5 & 2 & 1 & 3 & 8 & 10 & 7 & 6 & 9 & 12 & 13 & 11 & 14 & 17 & 16 & 15 \\
\hline 12 & 1 & 10 & 3 & 9 & 4 & 2 & 5 & 7 & 8 & 6 & 14 & 12 & 11 & 17 & 15 & 13 & 16 \\
\hline 13 & 1 & 3 & 5 & 2 & 6 & 10 & 11 & 9 & 4 & 8 & 7 & 14 & 12 & 13 & 15 & 17 & 16 \\
\hline 14 & 5 & 7 & 4 & 1 & 2 & 8 & 9 & 10 & 6 & 3 & 13 & 11 & 12 & 16 & 15 & 17 & 14 \\
\hline 15 & 4 & 5 & 1 & 2 & 3 & 6 & 8 & 7 & 11 & 9 & 10 & 14 & 17 & 12 & 13 & 15 & 16 \\
\hline avg & $\begin{array}{c}2.8 \\
\pm 0.44\end{array}$ & $\begin{array}{c}4.3 \\
\pm 0.6\end{array}$ & $\begin{array}{l}4.8 \\
\pm 1\end{array}$ & $\begin{array}{c}5.3 \\
\pm 1.2\end{array}$ & $\begin{array}{c}5.5 \\
\pm 1.1\end{array}$ & $\begin{array}{c}6.5 \\
\pm 0.55\end{array}$ & $\begin{array}{c}7.7 \\
\pm 0.78\end{array}$ & $\begin{array}{c}7.7 \\
\pm 0.58\end{array}$ & $\begin{array}{l}8.1 \\
\pm 1\end{array}$ & $\begin{array}{c}8.7 \\
\pm 0.81\end{array}$ & $\begin{array}{c}11 \\
\pm 0.62\end{array}$ & $\begin{array}{c}11 \\
\pm 1\end{array}$ & $\begin{array}{c}13 \\
\pm 0.69\end{array}$ & $\begin{array}{c}14 \\
\pm 0.8\end{array}$ & $\begin{array}{c}14 \\
\pm 0.64\end{array}$ & $\begin{array}{c}15 \\
\pm 0.75\end{array}$ & $\begin{array}{c}15 \\
\pm 0.66\end{array}$ \\
\hline unimodal & 3 & 3 & 4.5 & 6 & 4.5 & 6 & 6.5 & 9 & 11 & 10 & 11 & 7.5 & 11 & 15 & 14 & 14 & 17 \\
\hline multimodal & 2.8 & 4.5 & 4.8 & 5.2 & 5.7 & 6.5 & 7.8 & 7.5 & 7.6 & 8.5 & 11 & 12 & 13 & 14 & 14 & 15 & 15 \\
\hline
\end{tabular}

TABLE I: Ranks of each algorithm for each test case, in dimension 10 and 30, for criteria worst.

[18] J. Nelder and R. Mead, "A simplex method for function minimization," Computer Journal 7, pp. 308-311, 1965.

[19] J. Kennedy and R. C. Eberhart, "Particle swarm optimization," in Proceedings of the IEEE International Conference on Neural Networks, 1995, pp. 1942-1948.

[20] Y. Shi and R. C. Eberhart, "A Modified Particle Swarm Optimizer," in Proceedings of IEEE International Conference on Evolutionary Computation. Washington, DC, USA: IEEE Computer Society, May 1998, pp. 69-73.

[21] S. Gelly, J. Mary, and O. Teytaud, "On the ultimate convergence rates for isotropic algorithms and the best choices among various forms of isotropy," in $10^{\text {th }}$ International Conference on Parallel Problem Solving from Nature (PPSN 2006), 2006. 


\begin{tabular}{|c|c|c|c|c|c|c|c|c|c|c|c|c|c|c|c|c|c|}
\hline \multicolumn{18}{|c|}{ Dim 10 , median } \\
\hline & CMA/CMA & $\begin{array}{l}\text { NM/ } \\
\text { NM } \\
\text { /NM }\end{array}$ & $\begin{array}{l}\text { CMA/ } \\
\text { CMA } \\
\text { /CMA }\end{array}$ & NM & $\begin{array}{l}\mathrm{DE} / \\
\mathrm{DE} \\
/ \mathrm{DE}\end{array}$ & $\begin{array}{l}\mathrm{NM} / \\
\mathrm{DE} \\
\text { /PSO }\end{array}$ & CMA & $\mathrm{DE}$ & $\begin{array}{l}\text { CMSA/ } \\
\text { NM } \\
\text { /PSO }\end{array}$ & $\begin{array}{c}\text { CMSA/ } \\
\text { DE } \\
\text { /PSO }\end{array}$ & SAiso & SA & $\begin{array}{l}\mathrm{PSO} / \\
\mathrm{PSO} \\
\text { /PSO }\end{array}$ & $\begin{array}{l}\text { CMSA/ } \\
\text { CMSA } \\
\text { /CMSA }\end{array}$ & SAcov & CMSA & PSO \\
\hline 1 & 1 & 2 & 4 & 6 & 9 & 10 & 3 & 11 & 5 & 7 & 8 & 16 & 13 & 14 & 17 & 12 & 15 \\
\hline 2 & 13 & 1 & 6 & 3 & 4 & 2 & 9 & 7 & 16 & 12 & 11 & 5 & 17 & 14 & 8 & 15 & 10 \\
\hline 3 & 3 & 9 & 2 & 4 & 6 & 10 & 1 & 7 & 5 & 14 & 8 & 15 & 13 & 11 & 12 & 17 & 16 \\
\hline 4 & 3 & 2 & 10 & 7 & 4 & 1 & 16 & 6 & 9 & 11 & 15 & 5 & 14 & 17 & 13 & 8 & 12 \\
\hline 5 & 1 & 3 & 13 & 2 & 7 & 5 & 16 & 10 & 14 & 4 & 17 & 6 & 15 & 9 & 12 & 8 & 11 \\
\hline 6 & 1 & 3 & 5 & 8 & 9 & 10 & 6 & 4 & 2 & 11 & 7 & 13 & 16 & 15 & 14 & 12 & 17 \\
\hline 7 & 6 & 1 & 2 & 7 & 9 & 4 & 5 & 10 & 3 & 14 & 8 & 12 & 11 & 15 & 16 & 13 & 17 \\
\hline 8 & 4 & 3 & 5 & 1 & 7 & 9 & 2 & 10 & 6 & 12 & 8 & 17 & 14 & 11 & 15 & 16 & 13 \\
\hline 9 & 9 & 16 & 13 & 17 & 2 & 5 & 1 & 7 & 14 & 11 & 10 & 6 & 4 & 12 & 3 & 15 & 8 \\
\hline 10 & 2 & 11 & 10 & 1 & 7 & 4 & 5 & 6 & 3 & 14 & 16 & 13 & 9 & 8 & 15 & 12 & 17 \\
\hline 11 & 2 & 4 & 1 & 5 & 10 & 6 & 3 & 8 & 7 & 14 & 17 & 9 & 12 & 11 & 13 & 15 & 16 \\
\hline 12 & 5 & 3 & 4 & 6 & 2 & 1 & 13 & 10 & 11 & 8 & 9 & 16 & 12 & 17 & 14 & 15 & 7 \\
\hline 13 & 5 & 1 & 2 & 3 & 6 & 9 & 4 & 12 & 10 & 13 & 8 & 17 & 7 & 11 & 15 & 14 & 16 \\
\hline 14 & 6 & 8 & 1 & 4 & 5 & 2 & 7 & 3 & 12 & 13 & 10 & 15 & 16 & 11 & 9 & 14 & 17 \\
\hline 15 & 5 & 7 & 3 & 11 & 2 & 13 & 4 & 10 & 15 & 1 & 8 & 12 & 6 & 9 & 14 & 16 & 17 \\
\hline avg & $\begin{array}{c}4.4 \\
\pm 0.85\end{array}$ & $\begin{array}{c}4.9 \\
\pm 1.1\end{array}$ & $\begin{array}{c}5.4 \\
\pm 1.1\end{array}$ & $\begin{array}{c}5.7 \\
\pm 1.1\end{array}$ & $\begin{array}{c}5.9 \\
\pm 0.7\end{array}$ & $\begin{array}{c}6.1 \\
\pm 0.99\end{array}$ & $\begin{array}{c}6.3 \\
\pm 1.3\end{array}$ & $\begin{array}{c}8.1 \\
\pm 0.68\end{array}$ & $\begin{array}{c}8.8 \\
\pm 1.2\end{array}$ & $\begin{array}{c}11 \\
\pm 1\end{array}$ & $\begin{array}{c}11 \\
\pm 0.94\end{array}$ & $\begin{array}{c}12 \\
\pm 1.2\end{array}$ & $\begin{array}{c}12 \\
\pm 1\end{array}$ & $\begin{array}{c}12 \\
\pm 0.73\end{array}$ & $\begin{array}{c}13 \\
\pm 0.93\end{array}$ & $\begin{array}{c}13 \\
\pm 0.7\end{array}$ & $\begin{array}{c}14 \\
\pm 0.91\end{array}$ \\
\hline unimodal & 7 & 1.5 & 5 & 4.5 & 6.5 & 6 & 6 & 9 & 10 & 9.5 & 9.5 & 10 & 15 & 14 & 12 & 14 & 12 \\
\hline multimodal & 4 & 5.5 & 5.5 & 5.8 & 5.8 & 6.1 & 6.4 & 7.9 & 8.5 & 11 & 11 & 12 & 11 & 12 & 13 & 13 & 14 \\
\hline \multicolumn{18}{|c|}{ Dim 30, median } \\
\hline & $\begin{array}{l}\mathrm{NM} / \\
\mathrm{NM} \\
\text { /NM }\end{array}$ & NM & CMA/CMA & CMA & $\begin{array}{l}\text { CMA/ } \\
\text { CMA } \\
\text { /CMA }\end{array}$ & $\begin{array}{c}\mathrm{NM} / \\
\mathrm{DE} \\
/ \mathrm{PSO}\end{array}$ & $\begin{array}{c}\text { CMSA/ } \\
\text { NM } \\
\text { /PSO }\end{array}$ & $\mathrm{DE}$ & $\begin{array}{l}\mathrm{DE} / \\
\mathrm{DE} \\
/ \mathrm{DE}\end{array}$ & $\begin{array}{c}\text { CMSA/ } \\
\text { DE } \\
/ \mathrm{PSO}\end{array}$ & SAiso & PSO & $\begin{array}{l}\mathrm{PSO} / \\
\mathrm{PSO} \\
\text { /PSO }\end{array}$ & CMSA & $\begin{array}{c}\text { CMSA/ } \\
\text { CMSA } \\
\text { /CMSA }\end{array}$ & SA & SAcov \\
\hline 1 & 6 & 4 & 1 & 3 & 2 & 7 & 5 & 11 & 10 & 9 & 8 & 12 & 13 & 15 & 14 & 16 & 17 \\
\hline 2 & 2 & 1 & 12 & 7 & 6 & 5 & 11 & 4 & 8 & 10 & 15 & 3 & 9 & 16 & 14 & 13 & 17 \\
\hline 3 & 4 & 7 & 3 & 1 & 2 & 5 & 6 & 9 & 8 & 10 & 15 & 13 & 16 & 12 & 17 & 11 & 14 \\
\hline 4 & 1 & 3 & 9 & 2 & 8 & 4 & 7 & 5 & 6 & 15 & 10 & 11 & 16 & 13 & 12 & 17 & 14 \\
\hline 5 & 1 & 3 & 16 & 15 & 17 & 4 & 2 & 10 & 5 & 6 & 12 & 8 & 7 & 13 & 14 & 9 & 11 \\
\hline 6 & 4 & 1 & 3 & 6 & 2 & 5 & 7 & 10 & 9 & 11 & 8 & 13 & 12 & 17 & 16 & 15 & 14 \\
\hline 7 & 3 & 7 & 2 & 4 & 5 & 1 & 6 & 9 & 8 & 10 & 14 & 11 & 12 & 15 & 13 & 16 & 17 \\
\hline 8 & 4 & 6 & 3 & 2 & 1 & 8 & 5 & 9 & 7 & 10 & 11 & 14 & 13 & 16 & 15 & 17 & 12 \\
\hline 9 & 3 & 6 & 9 & 13 & 16 & 4 & 7 & 1 & 12 & 10 & 11 & 2 & 5 & 15 & 17 & 14 & 8 \\
\hline 10 & 1 & 2 & 5 & 4 & 3 & 7 & 12 & 9 & 6 & 8 & 10 & 15 & 13 & 11 & 17 & 14 & 16 \\
\hline 11 & 5 & 4 & 3 & 1 & 2 & 9 & 6 & 10 & 7 & 8 & 12 & 13 & 14 & 16 & 11 & 15 & 17 \\
\hline 12 & 4 & 2 & 1 & 7 & 5 & 3 & 10 & 8 & 9 & 6 & 13 & 11 & 12 & 15 & 16 & 14 & 17 \\
\hline 13 & 2 & 1 & 3 & 5 & 6 & 8 & 4 & 11 & 10 & 9 & 7 & 14 & 12 & 13 & 15 & 17 & 16 \\
\hline 14 & 3 & 7 & 2 & 6 & 1 & 8 & 4 & 9 & 11 & 5 & 10 & 12 & 13 & 16 & 15 & 17 & 14 \\
\hline 15 & 4 & 5 & 2 & 1 & 3 & 6 & 7 & 9 & 10 & 11 & 8 & 16 & 17 & 13 & 12 & 14 & 15 \\
\hline avg & $\begin{array}{c}3.1 \\
\pm 0.39 \\
\end{array}$ & $\begin{array}{c}3.9 \\
\pm 0.59 \\
\end{array}$ & $\begin{array}{r}4.9 \\
\pm 1.2 \\
\end{array}$ & $\begin{array}{c}5.1 \\
\pm 1.1 \\
\end{array}$ & $\begin{array}{c}5.3 \\
\pm 1.3\end{array}$ & $\begin{array}{c}5.6 \\
\pm 0.58 \\
\end{array}$ & $\begin{array}{c}6.6 \\
\pm 0.7 \\
\end{array}$ & $\begin{array}{c}8.3 \\
\pm 0.72\end{array}$ & $\begin{array}{c}8.4 \\
\pm 0.51 \\
\end{array}$ & $\begin{array}{c}9.2 \\
\pm 0.63 \\
\end{array}$ & $\begin{array}{c}11 \\
\pm 0.67 \\
\end{array}$ & $\begin{array}{c}11 \\
\pm 1\end{array}$ & $\begin{array}{c}12 \\
\pm 0.84\end{array}$ & $\begin{array}{c}14 \\
\pm 0.46 \\
\end{array}$ & $\begin{aligned} & 15 \\
\pm & 0.5\end{aligned}$ & $\begin{array}{c}15 \\
\pm 0.6\end{array}$ & $\begin{array}{c}15 \\
\pm 0.68 \\
\end{array}$ \\
\hline unimodal & 4 & 2.5 & 6.5 & 5 & 4 & 6 & 8 & 7.5 & 9 & 9.5 & 12 & 7.5 & 11 & 16 & 14 & 14 & 17 \\
\hline multimodal & 3 & 4.2 & 4.7 & 5.2 & 5.5 & 5.5 & 6.4 & 8.4 & 8.3 & 9.2 & 11 & 12 & 12 & 14 & 15 & 15 & 14 \\
\hline
\end{tabular}

TABLE II: Ranks of each algorithm for each test case, in dimension 10 and 30, for criteria median. 


\begin{tabular}{|c|c|c|c|c|c|c|c|c|c|c|c|c|c|c|c|c|c|}
\hline \multicolumn{18}{|c|}{ Dim 10, mean } \\
\hline & CMA/CMA & $\begin{array}{l}\text { CMA/ } \\
\text { CMA } \\
\text { /CMA }\end{array}$ & NM & $\begin{array}{l}\mathrm{NM} / \\
\mathrm{NM} \\
\text { /NM }\end{array}$ & $\begin{array}{c}\mathrm{NM} / \\
\mathrm{DE} \\
/ \mathrm{PSO}\end{array}$ & $\begin{array}{l}\mathrm{DE} / \\
\mathrm{DE} \\
/ \mathrm{DE}\end{array}$ & CMA & $\mathrm{DE}$ & $\begin{array}{l}\text { CMSA/ } \\
\text { NM } \\
\text { /PSO }\end{array}$ & $\begin{array}{c}\text { CMSA/ } \\
\text { DE } \\
/ \mathrm{PSO}\end{array}$ & SAiso & $\begin{array}{l}\mathrm{PSO} / \\
\mathrm{PSO} \\
\text { /PSO }\end{array}$ & $\begin{array}{l}\text { CMSA/ } \\
\text { CMSA } \\
\text { /CMSA }\end{array}$ & SA & SAcov & CMSA & PSO \\
\hline 1 & 1 & 3 & 5 & 4 & 10 & 9 & 2 & 11 & 6 & 7 & 8 & 13 & 14 & 16 & 17 & 12 & 15 \\
\hline 2 & 11 & 5 & 3 & 1 & 2 & 4 & 8 & 6 & 15 & 10 & 14 & 17 & 12 & 16 & 9 & 13 & 7 \\
\hline 3 & 3 & 2 & 4 & 10 & 9 & 6 & 1 & 7 & 5 & 14 & 8 & 13 & 11 & 15 & 12 & 17 & 16 \\
\hline 4 & 3 & 10 & 7 & 2 & 1 & 4 & 16 & 6 & 9 & 11 & 15 & 14 & 17 & 5 & 13 & 8 & 12 \\
\hline 5 & 4 & 13 & 1 & 2 & 5 & 7 & 15 & 10 & 14 & 3 & 17 & 16 & 11 & 6 & 12 & 9 & 8 \\
\hline 6 & 1 & 5 & 8 & 4 & 10 & 9 & 6 & 3 & 2 & 11 & 7 & 16 & 15 & 13 & 14 & 12 & 17 \\
\hline 7 & 6 & 2 & 7 & 1 & 4 & 9 & 5 & 10 & 3 & 14 & 8 & 11 & 15 & 12 & 16 & 13 & 17 \\
\hline 8 & 3 & 4 & 1 & 5 & 9 & 7 & 2 & 10 & 6 & 12 & 8 & 14 & 11 & 17 & 15 & 16 & 13 \\
\hline 9 & 9 & 13 & 17 & 16 & 2 & 3 & 1 & 7 & 14 & 11 & 10 & 5 & 12 & 6 & 4 & 15 & 8 \\
\hline 10 & 2 & 9 & 1 & 10 & 3 & 6 & 4 & 5 & 13 & 14 & 16 & 8 & 7 & 12 & 15 & 11 & 17 \\
\hline 11 & 1 & 2 & 5 & 4 & 6 & 10 & 3 & 8 & 7 & 12 & 17 & 11 & 13 & 9 & 15 & 14 & 16 \\
\hline 12 & 5 & 4 & 6 & 2 & 1 & 3 & 12 & 10 & 13 & 8 & 9 & 11 & 17 & 16 & 14 & 15 & 7 \\
\hline 13 & 4 & 2 & 3 & 1 & 9 & 6 & 5 & 12 & 10 & 13 & 8 & 7 & 11 & 17 & 15 & 14 & 16 \\
\hline 14 & 6 & 2 & 4 & 8 & 1 & 5 & 7 & 3 & 12 & 13 & 10 & 16 & 11 & 15 & 9 & 14 & 17 \\
\hline 15 & 5 & 3 & 9 & 11 & 12 & 1 & 4 & 10 & 14 & 2 & 7 & 6 & 8 & 13 & 15 & 16 & 17 \\
\hline avg & $\begin{array}{c}4.3 \\
\pm 0.75\end{array}$ & $\begin{array}{l}5.3 \\
\pm 1\end{array}$ & $\begin{array}{c}5.4 \\
\pm 1.1\end{array}$ & $\begin{array}{c}5.4 \\
\pm 1.2\end{array}$ & $\begin{array}{l}5.6 \\
\pm 1\end{array}$ & $\begin{array}{c}5.9 \\
\pm 0.68\end{array}$ & $\begin{array}{c}6.1 \\
\pm 1.2\end{array}$ & $\begin{array}{c}7.9 \\
\pm 0.74\end{array}$ & $\begin{array}{c}9.5 \\
\pm 1.1\end{array}$ & $\begin{array}{c}10 \\
\pm 0.98\end{array}$ & $\begin{array}{c}11 \\
\pm 0.99\end{array}$ & $\begin{array}{c}12 \\
\pm 1\end{array}$ & $\begin{array}{c}12 \\
\pm 0.75\end{array}$ & $\begin{array}{c}13 \\
\pm 1.1\end{array}$ & $\begin{array}{c}13 \\
\pm 0.88\end{array}$ & $\begin{array}{c}13 \\
\pm 0.66\end{array}$ & $\begin{array}{c}14 \\
\pm 1\end{array}$ \\
\hline unimodal & 6 & 4 & 4 & 2.5 & 6 & 6.5 & 5 & 8.5 & 10 & 8.5 & 11 & 15 & 13 & 16 & 13 & 12 & 11 \\
\hline multimodal & 4 & 5.5 & 5.6 & 5.8 & 5.5 & 5.8 & 6.2 & 7.8 & 9.4 & 11 & 11 & 11 & 12 & 12 & 13 & 13 & 14 \\
\hline \multicolumn{18}{|c|}{ Dim 30, mean } \\
\hline & $\begin{array}{l}\mathrm{NM} / \\
\mathrm{NM} \\
\text { /NM }\end{array}$ & NM & CMA & $\begin{array}{l}\text { CMA/ } \\
\text { CMA } \\
\text { /CMA }\end{array}$ & CMA/CMA & $\begin{array}{c}\mathrm{NM} / \\
\mathrm{DE} \\
/ \mathrm{PSO}\end{array}$ & $\begin{array}{c}\text { CMSA/ } \\
\text { NM } \\
\text { /PSO }\end{array}$ & $\begin{array}{l}\mathrm{DE} / \\
\mathrm{DE} \\
/ \mathrm{DE}\end{array}$ & $\mathrm{DE}$ & $\begin{array}{c}\text { CMSA/ } \\
\text { DE } \\
/ \mathrm{PSO}\end{array}$ & PSO & SAiso & $\begin{array}{l}\mathrm{PSO} / \\
\mathrm{PSO} \\
\text { /PSO }\end{array}$ & CMSA & $\begin{array}{l}\text { CMSA/ } \\
\text { CMSA } \\
\text { /CMSA }\end{array}$ & SAcov & SA \\
\hline 1 & 5 & 4 & 3 & 2 & 1 & 7 & 6 & 9 & 11 & 10 & 12 & 8 & 13 & 15 & 14 & 17 & 16 \\
\hline 2 & 2 & 1 & 6 & 7 & 11 & 5 & 12 & 8 & 4 & 10 & 3 & 15 & 9 & 16 & 14 & 17 & 13 \\
\hline 3 & 4 & 7 & 1 & 2 & 3 & 6 & 5 & 8 & 9 & 10 & 15 & 14 & 16 & 11 & 17 & 12 & 13 \\
\hline 4 & 1 & 2 & 7 & 6 & 8 & 4 & 9 & 5 & 3 & 15 & 11 & 10 & 16 & 13 & 12 & 14 & 17 \\
\hline 5 & 1 & 2 & 15 & 16 & 17 & 3 & 4 & 5 & 11 & 8 & 6 & 13 & 7 & 9 & 10 & 14 & 12 \\
\hline 6 & 4 & 1 & 5 & 2 & 3 & 7 & 6 & 9 & 11 & 10 & 13 & 8 & 12 & 16 & 17 & 14 & 15 \\
\hline 7 & 5 & 6 & 3 & 2 & 1 & 7 & 4 & 8 & 9 & 10 & 11 & 14 & 12 & 15 & 13 & 17 & 16 \\
\hline 8 & 4 & 5 & 1 & 3 & 2 & 8 & 6 & 7 & 9 & 10 & 13 & 11 & 12 & 16 & 14 & 15 & 17 \\
\hline 9 & 2 & 5 & 10 & 15 & 13 & 4 & 6 & 11 & 1 & 9 & 3 & 12 & 7 & 17 & 14 & 8 & 16 \\
\hline 10 & 1 & 2 & 4 & 3 & 5 & 7 & 12 & 6 & 9 & 8 & 13 & 10 & 14 & 11 & 17 & 16 & 15 \\
\hline 11 & 5 & 4 & 2 & 1 & 3 & 8 & 6 & 7 & 10 & 9 & 11 & 13 & 12 & 15 & 14 & 16 & 17 \\
\hline 12 & 4 & 3 & 8 & 5 & 1 & 2 & 7 & 9 & 10 & 6 & 11 & 13 & 12 & 17 & 15 & 16 & 14 \\
\hline 13 & 2 & 1 & 5 & 6 & 3 & 8 & 4 & 10 & 11 & 9 & 14 & 7 & 12 & 13 & 15 & 16 & 17 \\
\hline 14 & 5 & 7 & 3 & 1 & 2 & 8 & 6 & 11 & 9 & 4 & 12 & 10 & 13 & 15 & 16 & 14 & 17 \\
\hline 15 & 4 & 5 & 1 & 3 & 2 & 6 & 7 & 10 & 9 & 11 & 16 & 8 & 17 & 12 & 13 & 15 & 14 \\
\hline avg & $\begin{array}{c}3.3 \\
\pm 0.41\end{array}$ & $\begin{array}{c}3.7 \\
\pm 0.55\end{array}$ & $\begin{array}{l}4.9 \\
\pm 1\end{array}$ & $\begin{array}{c}4.9 \\
\pm 1.2\end{array}$ & $\begin{array}{c}5 \\
+1.3\end{array}$ & $\begin{array}{c}6 \\
\pm 0.51\end{array}$ & $\begin{array}{c}6.7 \\
+0.65\end{array}$ & $\begin{array}{c}8.2 \\
\pm 0.5\end{array}$ & $\begin{array}{c}8.4 \\
\pm 0.81\end{array}$ & $\begin{array}{c}9.3 \\
\pm 0.62\end{array}$ & $\begin{array}{l}11 \\
\pm 1\end{array}$ & $\begin{array}{c}11 \\
\pm 0.67\end{array}$ & $\begin{array}{c}12 \\
+0.76\end{array}$ & $\begin{array}{c}14 \\
\pm 0.63\end{array}$ & $\begin{array}{c}14 \\
\pm 0.5\end{array}$ & $\begin{array}{c}15 \\
\pm 0.61\end{array}$ & $\begin{array}{c}15 \\
\pm 0.44\end{array}$ \\
\hline unimodal & 3.5 & 2.5 & 4.5 & 4.5 & 6 & 6 & 9 & 8.5 & 7.5 & 10 & 7.5 & 12 & 11 & 16 & 14 & 17 & 14 \\
\hline multimodal & 3.2 & 3.8 & 5 & 5 & 4.8 & 6 & 6.3 & 8.2 & 8.5 & 9.2 & 11 & 11 & 12 & 14 & 14 & 14 & 15 \\
\hline
\end{tabular}

TABLE III: Ranks of each algorithm for each test case, in dimension 10 and 30, for criteria mean. 


\begin{tabular}{|c|c|c|c|c|c|c|c|c|}
\hline \multicolumn{9}{|c|}{ Dim 10, best } \\
\hline & CMABIQR & $\mathrm{CMA}+\mathrm{QR}$ & CMA+BISMQR & CMABIMSQR & CMABI & CMABIMS & CMA+ & CMABI+MS \\
\hline 1 & 2 & 1 & 3 & 4 & 7 & 6 & 5 & 8 \\
\hline 2 & 2 & 1 & 3 & 4 & 5 & 7 & 8 & 6 \\
\hline 3 & 4 & 1 & 5 & 6 & 2 & 8 & 7 & 3 \\
\hline 4 & 4 & 1 & 5 & 6 & 2 & 7 & 8 & 3 \\
\hline 5 & 2 & 1 & 3 & 4 & 6 & 5 & 8 & 7 \\
\hline 6 & 1 & 4 & 2 & 3 & 6 & 5 & 8 & 7 \\
\hline 7 & 2 & 1 & 3 & 4 & 6 & 8 & 5 & 7 \\
\hline 8 & 1 & 4 & 2 & 3 & 5 & 6 & 7 & 8 \\
\hline 9 & 2 & 1 & 3 & 4 & 7 & 6 & 5 & 8 \\
\hline 10 & 1 & 5 & 2 & 3 & 4 & 6 & 7 & 8 \\
\hline 11 & 1 & 4 & 2 & 3 & 7 & 6 & 5 & 8 \\
\hline 12 & 1 & 4 & 2 & 3 & 6 & 5 & 8 & 7 \\
\hline 13 & 1 & 4 & 2 & 3 & 5 & 6 & 7 & 8 \\
\hline 14 & 2 & 1 & 3 & 4 & 6 & 5 & 8 & 7 \\
\hline 15 & 3 & 1 & 4 & 5 & 7 & 2 & 6 & 8 \\
\hline avg & $\begin{array}{c}1.9 \\
\pm 0.27\end{array}$ & $\begin{array}{c}2.3 \\
\pm 0.42\end{array}$ & $\begin{array}{c}2.9 \\
\pm 0.27\end{array}$ & $\begin{array}{c}3.9 \\
\pm 0.27\end{array}$ & $\begin{array}{c}5.4 \\
\pm 0.42\end{array}$ & $\begin{array}{c}5.9 \\
\pm 0.38\end{array}$ & $\begin{array}{c}6.8 \\
\pm 0.33\end{array}$ & $\begin{array}{c}6.9 \\
\pm 0.43\end{array}$ \\
\hline unimodal & 2 & 1 & 3 & 4 & 6 & 6.5 & 6.5 & 7 \\
\hline multimodal & 1.9 & 2.5 & 2.9 & 3.9 & 5.3 & 5.8 & 6.8 & 6.8 \\
\hline \multicolumn{9}{|c|}{ Dim 30, best } \\
\hline & CMABIQR & $\mathrm{CMA}+\mathrm{QR}$ & CMA+BISMQR & CMABIMSQR & CMABI & CMABIMS & CMABI+MS & CMA+ \\
\hline 1 & 1 & 4 & 2 & 3 & 7 & 6 & 8 & 5 \\
\hline 2 & 1 & 6 & 2 & 3 & 4 & 8 & 5 & 7 \\
\hline 3 & 2 & 1 & 3 & 4 & 6 & 8 & 7 & 5 \\
\hline 4 & 2 & 1 & 3 & 4 & 5 & 8 & 6 & 7 \\
\hline 5 & 1 & 4 & 2 & 3 & 6 & 5 & 7 & 8 \\
\hline 6 & 2 & 1 & 3 & 4 & 6 & 5 & 7 & 8 \\
\hline 7 & 3 & 2 & 4 & 5 & 6 & 1 & 7 & 8 \\
\hline 8 & 3 & 1 & 4 & 5 & 7 & 8 & 2 & 6 \\
\hline 9 & 2 & 1 & 3 & 4 & 7 & 5 & 8 & 6 \\
\hline 10 & 1 & 5 & 2 & 3 & 4 & 7 & 8 & 6 \\
\hline 11 & 3 & 1 & 4 & 5 & 2 & 8 & 6 & 7 \\
\hline 12 & 2 & 1 & 3 & 4 & 5 & 7 & 6 & 8 \\
\hline 13 & 1 & 5 & 2 & 3 & 6 & 4 & 7 & 8 \\
\hline 14 & 3 & 1 & 4 & 5 & 6 & 2 & 7 & 8 \\
\hline 15 & 5 & 1 & 6 & 7 & 3 & 8 & 4 & 2 \\
\hline avg & $\begin{array}{c}2.1 \\
\pm 0.29 \\
\end{array}$ & $\begin{array}{c}2.3 \\
\pm 0.48 \\
\end{array}$ & $\begin{array}{c}3.1 \\
\pm 0.29 \\
\end{array}$ & $\begin{array}{c}4.1 \\
\pm 0.29 \\
\end{array}$ & $\begin{array}{c}5.3 \\
\pm 0.39 \\
\end{array}$ & $\begin{array}{r}6 \\
\pm 0.59 \\
\end{array}$ & $\begin{array}{c}6.3 \\
\pm 0.42 \\
\end{array}$ & $\begin{array}{c}6.6 \\
\pm 0.43 \\
\end{array}$ \\
\hline unimodal & 1 & 5 & 2 & 3 & 5.5 & 7 & 6.5 & 6 \\
\hline multimodal & 2.3 & 1.9 & 3.3 & 4.3 & 5.3 & 5.8 & 6.3 & 6.7 \\
\hline
\end{tabular}

TABLE IV: Ranks of each CMA variant for each test case, in dimension 30, for criterion best. 


\begin{tabular}{|c|c|c|c|c|c|c|c|c|}
\hline \multicolumn{9}{|c|}{ Dim 10, worst } \\
\hline & CMABI & CMABIMS & CMA+ & CMABI+MS & CMABIQR & CMA+BISMQR & CMABIMSQR & $\mathrm{CMA}+\mathrm{QR}$ \\
\hline 1 & 6 & 1 & 2 & 7 & 3 & 4 & 5 & 8 \\
\hline 2 & 1 & 3 & 7 & 2 & 4 & 5 & 6 & 8 \\
\hline 3 & 1 & 4 & 5 & 2 & 6 & 7 & 8 & 3 \\
\hline 4 & 1 & 3 & 4 & 2 & 6 & 7 & 8 & 5 \\
\hline 5 & 2 & 1 & 7 & 3 & 4 & 5 & 6 & 8 \\
\hline 6 & 2 & 1 & 4 & 3 & 6 & 7 & 8 & 5 \\
\hline 7 & 4 & 3 & 1 & 2 & 5 & 6 & 7 & 8 \\
\hline 8 & 1 & 2 & 3 & 4 & 6 & 7 & 8 & 5 \\
\hline 9 & 3 & 2 & 1 & 4 & 5 & 6 & 7 & 8 \\
\hline 10 & 3 & 1 & 2 & 4 & 5 & 6 & 7 & 8 \\
\hline 11 & 2 & 1 & 4 & 3 & 5 & 6 & 7 & 8 \\
\hline 12 & 2 & 1 & 3 & 4 & 5 & 6 & 7 & 8 \\
\hline 13 & 2 & 7 & 1 & 3 & 4 & 5 & 6 & 8 \\
\hline 14 & 2 & 1 & 4 & 3 & 5 & 6 & 7 & 8 \\
\hline 15 & 2 & 4 & 1 & 3 & 5 & 6 & 7 & 8 \\
\hline avg & $\begin{array}{c}2.3 \\
\pm 0.34\end{array}$ & $\begin{array}{c}2.3 \\
\pm 0.44\end{array}$ & $\begin{array}{c}3.3 \\
\pm 0.52\end{array}$ & $\begin{array}{c}3.3 \\
\pm 0.33\end{array}$ & $\begin{array}{c}4.9 \\
\pm 0.23\end{array}$ & $\begin{array}{c}5.9 \\
\pm 0.23\end{array}$ & $\begin{array}{c}6.9 \\
\pm 0.23\end{array}$ & $\begin{array}{c}7.1 \\
\pm 0.43\end{array}$ \\
\hline unimodal & 3.5 & 2 & 4.5 & 4.5 & 3.5 & 4.5 & 5.5 & 8 \\
\hline multimodal & 2.1 & 2.4 & 3.1 & 3.1 & 5.2 & 6.2 & 7.2 & 6.9 \\
\hline \multicolumn{9}{|c|}{ Dim 30, worst } \\
\hline & CMABI & CMABI+MS & CMA+ & CMABIMS & CMABIQR & $\mathrm{CMA}+\mathrm{QR}$ & CMA+BISMQR & CMABIMSQR \\
\hline 1 & 7 & 8 & 2 & 6 & 3 & 1 & 4 & 5 \\
\hline 2 & 2 & 4 & 1 & 3 & 5 & 8 & 6 & 7 \\
\hline 3 & 3 & 4 & 1 & 2 & 5 & 8 & 6 & 7 \\
\hline 4 & 1 & 2 & 3 & 4 & 5 & 8 & 6 & 7 \\
\hline 5 & 1 & 2 & 3 & 4 & 5 & 8 & 6 & 7 \\
\hline 6 & 3 & 4 & 1 & 2 & 6 & 5 & 7 & 8 \\
\hline 7 & 1 & 2 & 3 & 4 & 5 & 8 & 6 & 7 \\
\hline 8 & 4 & 1 & 2 & 8 & 5 & 3 & 6 & 7 \\
\hline 9 & 2 & 3 & 4 & 1 & 6 & 5 & 7 & 8 \\
\hline 10 & 1 & 3 & 4 & 2 & 6 & 5 & 7 & 8 \\
\hline 11 & 1 & 2 & 3 & 4 & 5 & 8 & 6 & 7 \\
\hline 12 & 1 & 4 & 3 & 2 & 5 & 8 & 6 & 7 \\
\hline 13 & 3 & 2 & 4 & 1 & 5 & 8 & 6 & 7 \\
\hline 14 & 1 & 3 & 8 & 2 & 5 & 4 & 6 & 7 \\
\hline 15 & 2 & 3 & 7 & 8 & 4 & 1 & 5 & 6 \\
\hline avg & $\begin{array}{c}2.2 \\
\pm 0.43\end{array}$ & $\begin{array}{c}3.1 \\
\pm 0.42\end{array}$ & $\begin{array}{c}3.3 \\
\pm 0.52\end{array}$ & $\begin{array}{c}3.5 \\
\pm 0.58\end{array}$ & $\begin{array}{c}5 \\
\pm 0.2\end{array}$ & $\begin{array}{c}5.9 \\
\pm 0.68\end{array}$ & $\begin{array}{c}6 \\
+0.2\end{array}$ & $\begin{array}{c}7 \\
+0.2\end{array}$ \\
\hline unimodal & 4.5 & 6 & 1.5 & 4.5 & 4 & 4.5 & 5 & 6 \\
\hline multimodal & 1.8 & 2.7 & 3.5 & 3.4 & 5.2 & 6.1 & 6.2 & 7.2 \\
\hline
\end{tabular}

TABLE V: Ranks of each CMA variant for each test case, in dimension 30, for criterion worst. 


\begin{tabular}{|c|c|c|c|c|c|c|c|c|}
\hline \multicolumn{9}{|c|}{ Dim 10, median } \\
\hline & CMABIQR & CMABIMS & CMA+BISMQR & CMABI & $\mathrm{CMA}+\mathrm{QR}$ & CMA+ & CMABIMSQR & CMABI+MS \\
\hline 1 & 1 & 6 & 2 & 7 & 4 & 5 & 3 & 8 \\
\hline 2 & 3 & 7 & 4 & 1 & 6 & 8 & 5 & 2 \\
\hline 3 & 5 & 8 & 6 & 3 & 1 & 2 & 7 & 4 \\
\hline 4 & 4 & 3 & 5 & 1 & 7 & 8 & 6 & 2 \\
\hline 5 & 4 & 1 & 5 & 2 & 7 & 8 & 6 & 3 \\
\hline 6 & 1 & 5 & 2 & 6 & 4 & 8 & 3 & 7 \\
\hline 7 & 3 & 8 & 4 & 6 & 2 & 1 & 5 & 7 \\
\hline 8 & 4 & 2 & 5 & 1 & 8 & 3 & 6 & 7 \\
\hline 9 & 3 & 2 & 4 & 6 & 8 & 1 & 5 & 7 \\
\hline 10 & 3 & 1 & 4 & 6 & 8 & 2 & 5 & 7 \\
\hline 11 & 2 & 5 & 3 & 7 & 6 & 1 & 4 & 8 \\
\hline 12 & 3 & 1 & 4 & 6 & 2 & 8 & 5 & 7 \\
\hline 13 & 1 & 8 & 2 & 4 & 6 & 5 & 3 & 7 \\
\hline 14 & 4 & 1 & 5 & 2 & 3 & 8 & 6 & 7 \\
\hline 15 & 4 & 1 & 5 & 8 & 2 & 7 & 6 & 3 \\
\hline avg & $\begin{aligned} & 3 \\
& \pm 0.32 \\
&\end{aligned}$ & $\begin{array}{c}3.9 \\
\pm 0.75 \\
\end{array}$ & $\begin{aligned} & 4 \\
& \pm 0.32 \\
&\end{aligned}$ & $\begin{array}{c}4.4 \\
\pm 0.65 \\
\end{array}$ & $\begin{array}{c}4.9 \\
+0.64\end{array}$ & $\begin{aligned} & 5 \\
\pm & 0.78\end{aligned}$ & $\begin{aligned} & 5 \\
\pm & 0.32\end{aligned}$ & $\begin{array}{c}5.7 \\
\pm 0.57\end{array}$ \\
\hline unimodal & 2 & 6.5 & 3 & 4 & 5 & 6.5 & 4 & 5 \\
\hline multimodal & 3.2 & 3.5 & 4.2 & 4.5 & 4.9 & 4.8 & 5.2 & 5.8 \\
\hline \multicolumn{9}{|c|}{ Dim 30, median } \\
\hline & CMABIQR & CMA+BISMQR & $\mathrm{CMA}+\mathrm{QR}$ & CMABI & CMABIMS & CMABIMSQR & CMABI+MS & CMA+ \\
\hline 1 & 1 & 2 & 4 & 8 & 5 & 3 & 7 & 6 \\
\hline 2 & 1 & 2 & 8 & 5 & 7 & 3 & 6 & 4 \\
\hline 3 & 5 & 6 & 1 & 4 & 8 & 7 & 3 & 2 \\
\hline 4 & 3 & 4 & 7 & 1 & 8 & 5 & 6 & 2 \\
\hline 5 & 2 & 3 & 5 & 6 & 1 & 4 & 7 & 8 \\
\hline 6 & 3 & 4 & 2 & 7 & 1 & 5 & 8 & 6 \\
\hline 7 & 4 & 5 & 3 & 7 & 1 & 6 & 2 & 8 \\
\hline 8 & 3 & 4 & 2 & 6 & 8 & 5 & 1 & 7 \\
\hline 9 & 5 & 6 & 1 & 4 & 2 & 7 & 3 & 8 \\
\hline 10 & 3 & 4 & 8 & 1 & 6 & 5 & 7 & 2 \\
\hline 11 & 2 & 3 & 5 & 6 & 8 & 4 & 7 & 1 \\
\hline 12 & 2 & 3 & 5 & 6 & 7 & 4 & 1 & 8 \\
\hline 13 & 3 & 4 & 6 & 2 & 1 & 5 & 7 & 8 \\
\hline 14 & 5 & 6 & 3 & 2 & 1 & 7 & 4 & 8 \\
\hline 15 & 2 & 3 & 1 & 7 & 8 & 4 & 6 & 5 \\
\hline avg & $\begin{array}{c}2.9 \\
\pm 0.34\end{array}$ & $\begin{array}{c}3.9 \\
\pm 0.34\end{array}$ & $\begin{array}{c}4.1 \\
\pm 0.64\end{array}$ & $\begin{array}{c}4.8 \\
\pm 0.6\end{array}$ & $\begin{array}{c}4.8 \\
\pm 0.82\end{array}$ & $\begin{array}{c}4.9 \\
\pm 0.34\end{array}$ & $\begin{aligned} & 5 \\
\pm & 0.62\end{aligned}$ & $\begin{array}{c}5.5 \\
\pm 0.69\end{array}$ \\
\hline unimodal & 1 & 2 & 6 & 6.5 & 6 & 3 & 6.5 & 5 \\
\hline multimodal & 3.2 & 4.2 & 3.8 & 4.5 & 4.6 & 5.2 & 4.8 & 5.6 \\
\hline
\end{tabular}

TABLE VI: Ranks of each CMA variant for each test case, in dimension 30, for criterion median. 


\begin{tabular}{|c|c|c|c|c|c|c|c|c|}
\hline \multicolumn{9}{|c|}{ Dim 10, mean } \\
\hline & CMABIQR & CMABIMS & CMA+BISMQR & CMABI & CMABIMSQR & CMABI+MS & CMA+ & $\mathrm{CMA}+\mathrm{QR}$ \\
\hline 1 & 1 & 5 & 2 & 7 & 3 & 6 & 4 & 8 \\
\hline 2 & 3 & 6 & 4 & 1 & 5 & 2 & 8 & 7 \\
\hline 3 & 4 & 8 & 5 & 1 & 6 & 3 & 7 & 2 \\
\hline 4 & 5 & 3 & 6 & 1 & 7 & 2 & 8 & 4 \\
\hline 5 & 4 & 1 & 5 & 2 & 6 & 3 & 8 & 7 \\
\hline 6 & 2 & 1 & 3 & 6 & 4 & 7 & 8 & 5 \\
\hline 7 & 4 & 8 & 5 & 3 & 6 & 2 & 1 & 7 \\
\hline 8 & 4 & 2 & 5 & 1 & 6 & 7 & 3 & 8 \\
\hline 9 & 3 & 2 & 4 & 7 & 5 & 8 & 1 & 6 \\
\hline 10 & 3 & 1 & 4 & 6 & 5 & 7 & 2 & 8 \\
\hline 11 & 1 & 5 & 2 & 6 & 3 & 7 & 4 & 8 \\
\hline 12 & 2 & 1 & 3 & 5 & 4 & 6 & 8 & 7 \\
\hline 13 & 1 & 8 & 2 & 4 & 3 & 6 & 5 & 7 \\
\hline 14 & 4 & 1 & 5 & 2 & 6 & 7 & 8 & 3 \\
\hline 15 & 3 & 1 & 4 & 8 & 5 & 7 & 6 & 2 \\
\hline avg & $\begin{array}{c}2.9 \\
\pm 0.33\end{array}$ & $\begin{array}{c}3.5 \\
\pm 0.74\end{array}$ & $\begin{array}{c}3.9 \\
\pm 0.33\end{array}$ & $\begin{array}{c}4 \\
\pm 0.66\end{array}$ & $\begin{array}{c}4.9 \\
\pm 0.33\end{array}$ & $\begin{array}{c}5.3 \\
\pm 0.57\end{array}$ & $\begin{array}{c}5.4 \\
\pm 0.7\end{array}$ & $\begin{array}{c}5.9 \\
\pm 0.56\end{array}$ \\
\hline unimodal & 2 & 5.5 & 3 & 4 & 4 & 4 & 6 & 7.5 \\
\hline multimodal & 3.1 & 3.2 & 4.1 & 4 & 5.1 & 5.5 & 5.3 & 5.7 \\
\hline \multicolumn{9}{|c|}{ Dim 30, mean } \\
\hline & CMABIQR & CMA+BISMQR & CMABI & $\mathrm{CMA}+\mathrm{QR}$ & CMABIMS & CMABI+MS & CMABIMSQR & CMA+ \\
\hline 1 & 1 & 2 & 8 & 4 & 6 & 7 & 3 & 5 \\
\hline 2 & 2 & 3 & 1 & 8 & 7 & 6 & 4 & 5 \\
\hline 3 & 4 & 5 & 3 & 2 & 8 & 7 & 6 & 1 \\
\hline 4 & 4 & 5 & 1 & 7 & 8 & 3 & 6 & 2 \\
\hline 5 & 2 & 3 & 5 & 7 & 1 & 6 & 4 & 8 \\
\hline 6 & 3 & 4 & 6 & 2 & 1 & 8 & 5 & 7 \\
\hline 7 & 4 & 5 & 7 & 3 & 1 & 2 & 6 & 8 \\
\hline 8 & 3 & 4 & 7 & 2 & 8 & 1 & 5 & 6 \\
\hline 9 & 4 & 5 & 7 & 1 & 2 & 3 & 6 & 8 \\
\hline 10 & 4 & 5 & 1 & 8 & 3 & 7 & 6 & 2 \\
\hline 11 & 2 & 3 & 1 & 8 & 7 & 5 & 4 & 6 \\
\hline 12 & 4 & 5 & 1 & 3 & 7 & 2 & 6 & 8 \\
\hline 13 & 2 & 3 & 6 & 5 & 1 & 7 & 4 & 8 \\
\hline 14 & 5 & 6 & 2 & 3 & 1 & 4 & 7 & 8 \\
\hline 15 & 2 & 3 & 7 & 1 & 8 & 6 & 4 & 5 \\
\hline avg & $\begin{array}{c}3.1 \\
\pm 0.3 \\
\end{array}$ & $\begin{array}{c}4.1 \\
\pm 0.3 \\
\end{array}$ & $\begin{array}{c}4.2 \\
\pm 0.73 \\
\end{array}$ & $\begin{array}{c}4.3 \\
\pm 0.69 \\
\end{array}$ & $\begin{array}{c}4.6 \\
\pm 0.82\end{array}$ & $\begin{array}{c}4.9 \\
\pm 0.58 \\
\end{array}$ & $\begin{array}{c}5.1 \\
\pm 0.3\end{array}$ & $\begin{array}{c}5.8 \\
\pm 0.63 \\
\end{array}$ \\
\hline unimodal & 1.5 & 2.5 & 4.5 & 6 & 6.5 & 6.5 & 3.5 & 5 \\
\hline multimodal & 3.3 & 4.3 & 4.2 & 4 & 4.3 & 4.7 & 5.3 & 5.9 \\
\hline
\end{tabular}

TABLE VII: Ranks of each CMA variant for each test case, in dimension 30, for criterion mean. 


\begin{tabular}{|c|c|c|c|c|c|}
\hline F & Best & Worst & Median & Mean & Std \\
\hline 1 & 3066.821720 & 27350.414967 & 10303.331393 & 13532.288748 & 8015.631303 \\
2 & 17946.244786 & 75601.839181 & 38585.782508 & 39525.522486 & 15424.252191 \\
3 & 1.010692 & 15.066042 & 6.772117 & 7.342122 & 4.307489 \\
4 & 130.551411 & 2754.489295 & 2213.214380 & 1919.328740 & 798.520928 \\
5 & 0.428649 & 4.694434 & 3.378933 & 3.178128 & 1.239418 \\
6 & 0.257797 & 0.816413 & 0.500261 & 0.494573 & 0.153775 \\
7 & 0.288968 & 1.247096 & 0.495755 & 0.590027 & 0.302133 \\
8 & 1.621837 & 6.750625 & 4.885070 & 4.552976 & 1.573973 \\
9 & 3.353918 & 4.595982 & 4.222199 & 4.156896 & 0.340649 \\
10 & 11346.738112 & 2450865.764312 & 263107.148179 & 556046.674992 & 730726.425838 \\
11 & 2.187530 & 40.353662 & 6.419068 & 8.149008 & 7.903810 \\
12 & 31.308448 & 610.640458 & 307.249692 & 322.513038 & 153.876607 \\
13 & 316.642541 & 333.405878 & 320.124508 & 321.868712 & 4.217949 \\
14 & 186.334995 & 210.410137 & 202.610188 & 201.771257 & 6.009089 \\
15 & 10.511656 & 528.958620 & 408.019290 & 340.349673 & 170.162921 \\
\hline
\end{tabular}

TABLE VIII: Errors obtained by CMASPHI-QR in 10D

\begin{tabular}{|c|c|c|c|c|c|}
\hline $\mathrm{F}$ & Best & Worst & Median & Mean & Std \\
\hline 1 & 4410.546684 & 2433205.172072 & 9909.121145 & 203114.035298 & 611688.572924 \\
2 & 73838.778110 & 155921.584174 & 118489.287353 & 117191.061224 & 21987.789028 \\
3 & 13.348001 & 37.062026 & 20.819096 & 21.305606 & 5.062646 \\
4 & 2553.626647 & 9575.708781 & 8591.667368 & 7588.016926 & 2185.679036 \\
5 & 4.662556 & 6.673121 & 5.284304 & 5.437349 & 0.591955 \\
6 & 0.373039 & 1.045966 & 0.659812 & 0.685387 & 0.235695 \\
7 & 0.323589 & 1.313365 & 0.535025 & 0.636289 & 0.286018 \\
8 & 6.749165 & 56.118232 & 22.244325 & 22.911469 & 10.927353 \\
9 & 13.241557 & 14.225287 & 13.976680 & 13.848334 & 0.303010 \\
10 & 610222.482957 & 8220937.807046 & 2549366.836787 & 2774403.117855 & 2212879.888269 \\
11 & 17.995147 & 88.531156 & 22.161005 & 27.828859 & 19.823562 \\
12 & 224.026689 & 1078.687135 & 640.246067 & 671.996207 & 282.541119 \\
13 & 354.170628 & 400.510809 & 376.774443 & 377.075234 & 11.912947 \\
14 & 218.364928 & 271.850932 & 243.197289 & 245.710839 & 15.669614 \\
15 & 600.342406 & 887.127952 & 771.879600 & 758.132119 & 79.104475 \\
\hline
\end{tabular}

TABLE IX: Errors obtained by CMASPHI-QR in 30D

\begin{tabular}{|c|c|}
\hline Func. & $\hat{T}_{1} / T_{0}$ \\
\hline 1 & $5.20045 \mathrm{e}-01$ \\
2 & $2.61135 \mathrm{e}-01$ \\
3 & $1.05740 \mathrm{e}+00$ \\
4 & $2.81510 \mathrm{e}-01$ \\
5 & $5.36625 \mathrm{e}-01$ \\
6 & $3.41910 \mathrm{e}-01$ \\
7 & $3.59610 \mathrm{e}-01$ \\
8 & $3.36335 \mathrm{e}-01$ \\
9 & $2.66570 \mathrm{e}-01$ \\
10 & $2.80205 \mathrm{e}-01$ \\
11 & $4.34245 \mathrm{e}-01$ \\
12 & $3.10260 \mathrm{e}-01$ \\
13 & $2.94360 \mathrm{e}-01$ \\
14 & $3.13660 \mathrm{e}-01$ \\
15 & $1.13885 \mathrm{e}+00$ \\
\hline
\end{tabular}

TABLE X: Computational Complexity for best results in 10D

\begin{tabular}{|c|c|}
\hline Func. & $\hat{T}_{1} / T_{0}$ \\
\hline 1 & $1.25175 \mathrm{e}+00$ \\
2 & $1.17270 \mathrm{e}+00$ \\
3 & $8.35025 \mathrm{e}+00$ \\
4 & $1.27570 \mathrm{e}+00$ \\
5 & $3.27215 \mathrm{e}+00$ \\
6 & $1.22645 \mathrm{e}+00$ \\
7 & $1.24210 \mathrm{e}+00$ \\
8 & $1.29370 \mathrm{e}+00$ \\
9 & $1.15715 \mathrm{e}+00$ \\
10 & $1.46540 \mathrm{e}+00$ \\
11 & $2.71990 \mathrm{e}+00$ \\
12 & $1.56960 \mathrm{e}+00$ \\
13 & $1.54560 \mathrm{e}+00$ \\
14 & $1.67210 \mathrm{e}+00$ \\
15 & $8.83170 \mathrm{e}+00$ \\
\hline
\end{tabular}

TABLE XI: Computational Complexity for best results in 30D

\begin{tabular}{|c|c|c|c|c|}
\hline CMA Variants & Mean & STD & Median & Rank \\
\hline CMA+ & $5.78 \mathrm{e} 8$ & $1.60 \mathrm{e} 8$ & $5.34 \mathrm{e} 8$ & 20 \\
CMA+QR & $4.54 \mathrm{e} 8$ & $2.90 \mathrm{e} 7$ & $4.59 \mathrm{e} 8$ & 15 \\
CMABI+ & $5.99 \mathrm{e} 8$ & $1.81 \mathrm{e} 8$ & $5.41 \mathrm{e} 8$ & 22 \\
CMABIQR & $4.52 \mathrm{e} 8$ & $2.81 \mathrm{e} 7$ & $4.46 \mathrm{e} 8$ & 14 \\
CMABI & $6.17 \mathrm{e} 8$ & $1.94 \mathrm{e} 8$ & $5.80 \mathrm{e} 8$ & 24 \\
CMABISS+QR & $4.54 \mathrm{e} 8$ & $2.90 \mathrm{e} 7$ & $4.59 \mathrm{e} 8$ & 15 \\
CMABISS+ & $6.06 \mathrm{e} 8$ & $1.87 \mathrm{e} 8$ & $5.38 \mathrm{e} 8$ & 23 \\
CMABISSQR & $4.51 \mathrm{e} 8$ & $2.70 \mathrm{e} 7$ & $4.49 \mathrm{e} 8$ & 13 \\
CMABISS & $5.58 \mathrm{e} 8$ & $1.74 \mathrm{e} 8$ & $5.36 \mathrm{e} 8$ & 19 \\
CMAMI+ & $5.79 \mathrm{e} 8$ & $1.73 \mathrm{e} 8$ & $5.98 \mathrm{e} 8$ & 21 \\
CMAMI+QR & $4.55 \mathrm{e} 8$ & $2.83 \mathrm{e} 7$ & $4.59 \mathrm{e} 8$ & 18 \\
CMASPBI+ & $7.20 \mathrm{e} 7$ & $1.98 \mathrm{e} 7$ & $7.69 \mathrm{e} 7$ & 10 \\
CMASPBI+QR & $5.54 \mathrm{e} 7$ & $8.35 \mathrm{e} 6$ & $5.38 \mathrm{e} 7$ & 6 \\
CMABPBI+ & $5.39 \mathrm{e} 9$ & $6.23 \mathrm{e} 8$ & $5.40 \mathrm{e} 9$ & 27 \\
CMABPBI+QR & $4.30 \mathrm{e} 9$ & $1.98 \mathrm{e} 8$ & $4.24 \mathrm{e} 9$ & 25 \\
CMABPBI & $5.72 \mathrm{e} 9$ & $1.20 \mathrm{e} 9$ & $5.62 \mathrm{e} 9$ & 28 \\
CMABPBIQR & $4.30 \mathrm{e} 9$ & $1.98 \mathrm{e} 8$ & $4.24 \mathrm{e} 9$ & 25 \\
CMASPBI & $8.08 \mathrm{e} 7$ & $2.52 \mathrm{e} 7$ & $7.66 \mathrm{e} 7$ & 12 \\
CMASPBIQR & $5.21 \mathrm{e} 7$ & $3.47 \mathrm{e} 6$ & $5.20 \mathrm{e} 7$ & 1 \\
CMASPBISS+ & $7.39 \mathrm{e} 7$ & $2.66 \mathrm{e} 7$ & $6.42 \mathrm{e} 7$ & 11 \\
CMASPBISS+QR & $5.24 \mathrm{e} 7$ & $3.55 \mathrm{e} 6$ & $5.26 \mathrm{e} 7$ & 5 \\
CMASPBISS & $6.57 \mathrm{e} 7$ & $1.99 \mathrm{e} 7$ & $6.97 \mathrm{e} 7$ & 8 \\
CMASPBISS+QR & $5.21 \mathrm{e} 7$ & $3.47 \mathrm{e} 6$ & $5.20 \mathrm{e} 7$ & 1 \\
CMASPMI+ & $6.96 \mathrm{e} 7$ & $1.56 \mathrm{e} 7$ & $6.76 \mathrm{e} 7$ & 9 \\
CMASPMI+QR & $5.21 \mathrm{e} 7$ & $3.47 \mathrm{e} 6$ & $5.20 \mathrm{e} 7$ & 1 \\
CMASPHI+ & $6.53 \mathrm{e} 7$ & $1.80 \mathrm{e} 7$ & $6.34 \mathrm{e} 7$ & 7 \\
CMASPHI+QR & $5.21 \mathrm{e} 7$ & $3.47 \mathrm{e} 6$ & $5.20 \mathrm{e} 7$ & 1 \\
\hline
\end{tabular}

TABLE XII: Results of different variants of CMA-ES

\begin{tabular}{|c|c|c|c|c|}
\hline NM Variants & Mean & STD & Median & Rank \\
\hline$\alpha=1, \gamma=2, \rho=-.5, \sigma=.5$ & $1.11 \mathrm{e} 10$ & $5.16 \mathrm{e} 9$ & $1.00 \mathrm{e} 10$ & 2 \\
$\alpha=.8, \gamma=2, \rho=-.5, \sigma=.5$ & $3.27 \mathrm{e} 10$ & $1.16 \mathrm{e} 10$ & $2.98 \mathrm{e} 10$ & 5 \\
$\alpha=1, \gamma=2, \rho=-.75, \sigma=.5$ & $1.13 \mathrm{e} 10$ & $6.51 \mathrm{e} 9$ & $9.91 \mathrm{e} 9$ & 3 \\
$\alpha=1, \gamma=2, \rho=-.5, \sigma=.75$ & $1.06 \mathrm{e} 10$ & $5.41 \mathrm{e} 9$ & $9.72 \mathrm{e} 9$ & 1 \\
$\alpha=1.5, \gamma=3, \rho=-.25, \sigma=.25$ & $3.55 \mathrm{e} 10$ & $1.28 \mathrm{e} 10$ & $3.26 \mathrm{e} 10$ & 6 \\
$\alpha=.5, \gamma=1.5, \rho=-.75, \sigma=.75$ & $9.84 \mathrm{e} 10$ & $1.02 \mathrm{e} 10$ & $1.00 \mathrm{e} 11$ & 7 \\
\hline
\end{tabular}

TABLE XIII: Results of different variants of Nelder-Mead

\begin{tabular}{|c|c|c|c|c|}
\hline DE Variants & Mean & STD & Median & Rank \\
\hline $\begin{array}{c}D E / \text { curr }- \text { to }- \text { best } / 1 \\
f_{1}=.8, f_{2}=.8, \text { cr }=.5\end{array}$ & $7.17 \mathrm{e} 10$ & $8.69 \mathrm{e} 9$ & $7.14 \mathrm{e} 10$ & 8 \\
$D E /$ curr - to - best $/ 1$ & & & & \\
$f_{1}=1, f_{2}=1$, cr $=.8$ & $1.34 \mathrm{e} 11$ & $2.52 \mathrm{e} 10$ & $1.38 \mathrm{e} 11$ & 9 \\
$D E /$ curr - to - best $/ 1$ & & & & \\
$f_{1}=1, f_{2}=1$, cr $=.5$ & $6.72 \mathrm{e} 10$ & $1.29 \mathrm{e} 10$ & $6.96 \mathrm{e} 10$ & 1 \\
$D E /$ curr $-t o-$ best $/ 1$ & & & & \\
$f_{1}=.5, f_{2}=.8$, cr $=.5$ & $7.02 \mathrm{e} 10$ & $1.24 \mathrm{e} 10$ & $7.01 \mathrm{e} 10$ & 5 \\
$D E /$ rand - to - best $/ 1$ & & & & \\
$f_{1}=1, f_{2}=1$, cr $=.5$ & $7.14 \mathrm{e} 10$ & $1.12 \mathrm{e} 10$ & $6.99 \mathrm{e} 10$ & 6 \\
$D E /$ best $/ 1$ & & & & \\
$f_{1}=1, f_{2}=1$, cr $=.5$ & $6.95 \mathrm{e} 10$ & $9.39 \mathrm{e} 9$ & $6.97 \mathrm{e} 10$ & 4 \\
$D E /$ best $/ 1$ & & & & \\
$f_{1}=1, f_{2}=1$, cr $=.5$ & $6.94 \mathrm{e} 10$ & $9.55 \mathrm{e} 9$ & $6.97 \mathrm{e} 10$ & 3 \\
$D E /$ best $/ 1$ & & & & \\
$f_{1}=1, f_{2}=1$, cr $=.5$ & $6.94 \mathrm{e} 10$ & $9.54 \mathrm{e} 9$ & $6.97 \mathrm{e} 10$ & 2 \\
\hline
\end{tabular}

TABLE XIV: Results of different variants of Differential Evolution 


\begin{tabular}{|c|c|c|c|c|}
\hline PSO Variants & Mean & STD & Median & Rank \\
\hline 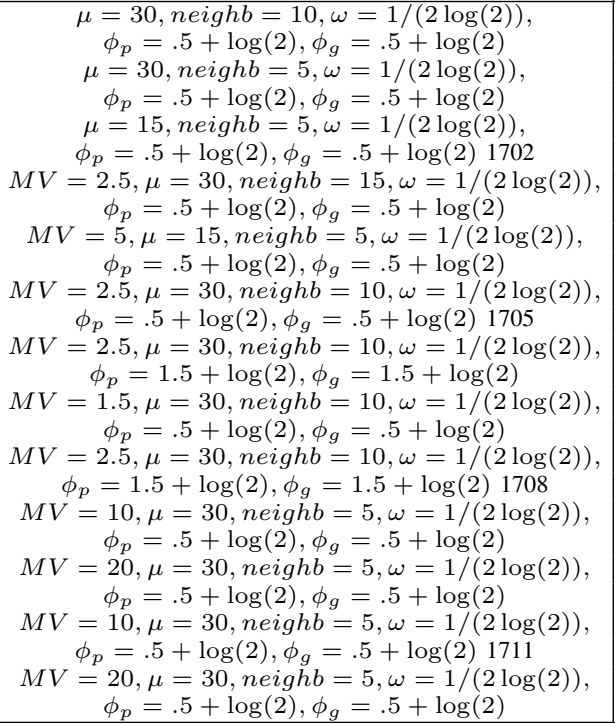 & $\begin{array}{l}1.43 \mathrm{e} 11 \\
1.11 \mathrm{e} 11 \\
5.63 \mathrm{e} 10 \\
4.45 \mathrm{e} 10 \\
5.89 \mathrm{e} 10 \\
4.64 \mathrm{e} 10\end{array}$ & $\begin{array}{l}1.22 \mathrm{e} 10 \\
1.13 \mathrm{e} 10 \\
8.16 \mathrm{e} 9 \\
1.34 \mathrm{e} 10 \\
1.27 \mathrm{e} 10 \\
1.49 \mathrm{e} 10\end{array}$ & $\begin{array}{l}1.11 \mathrm{e} 11 \\
1.16 \mathrm{e} 11 \\
1.45 \mathrm{e} 11 \\
1.11 \mathrm{e} 11 \\
5.77 \mathrm{e} 10 \\
4.60 \mathrm{e} 10 \\
5.50 \mathrm{e} 10 \\
4.70 \mathrm{e} 10\end{array}$ & $\begin{array}{l}12 \\
13\end{array}$ \\
\hline
\end{tabular}

TABLE XV: Results of different variants of PSO 